\title{
Análise das dosagens e concentrações séricas da ciclosporina A em cães da raça Golden Retriever normais ou afetados pela distrofia muscular
}

Adriana Caroprezo MORINI ${ }^{1}$ Maria Angélica MIGLINO ${ }^{1}$ Marileda Bonafim CARVALHO ${ }^{2}$ Daniele dos Santos MARTINS ${ }^{1}$ Carlos Eduardo AMBRÓSIO ${ }^{1}$ Lílian Jesus de OLIVEIRA ${ }^{1}$ Alida Abatemarco CATELLI ${ }^{1}$ Ana Paula SOUZA² José Roberto KFOURY JUNIOR ${ }^{1}$ Martha Maria Circhia Pinto LUPPI $^{1}$

Thaís Peixoto GAIAD ${ }^{1}$ Mayana ZATZ3

\section{Correspondência para:}

Adriana C. Morini - Av Prof Dr Orlando Marques de Paiva, 87 - São Paulo-SP 05507-000 -drimorini@usp.br

Recebido para publicação: 05/07/2004 Aprovado para publicação: 31/01/2008

\author{
1 - Faculdade de Medicina Veterinária e Zootecnia da Universidade de São Paulo, \\ São Paulo-SP \\ 2 - Faculdade de Ciências Agrárias e Veterinárias da Universidade Estadual \\ Paulista, Jaboticabal-SP \\ 3 - Instituto de Biologia da Universidade de São Paulo - Centro de Estudos do \\ Genoma Humano, São Paulo-SP
}

\section{Resumo}

A distrofia muscular dos cães Golden Retriever (GRMD), uma miopatia degenerativa causada pela ausência da distrofina é geneticamente homóloga à distrofia muscular de Duchenne que acomete humanos, portanto, estes cães são considerados modelos experimentais para estudos em terapia celular. Seu sucesso depende da imunossupressão adequada. A ciclosporina A (CsA) é indicada para tal e a monitorização de suas concentrações sangüíneas e efeitos adversos são essenciais para viabilizar a terapia. Foram estudados cães GRMD, e normais da mesma raça, submetidos a terapia com CsA, associada, nos GRMD, ao transplante de células tronco. Foram avaliados as concentrações sangüíneas do fármaco através de amostras coletadas a cada dois ou três dias e analisadas pelo método do imunoensaio por fluorescência (FPIA). Como resultado observamos que as concentrações de CsA oscilaram muito, em seis dos oito animais. Concluímos que as doses variam individualmente sendo de maior importância avaliar a concentração do fármaco no sangue e sua viabilização no uso da terapia celular.

\section{Introdução}

Terapias celulares que envolvem os transplantes trazem esperanças para o tratamento da Distrofia Muscular em humanos, no entanto enquanto a aplicação das mesmas não se torna viável, tratamentos com fármacos tornam-se cada vez mais testados. ${ }^{1}$ Atualmente as técnicas de transplantes heterólogos, são amplamente difundidas, sendo importante à queda da resposta imune. ${ }^{2}$ A ciclosporina A (CsA) é utilizada como imunossupressor em transplantes, como tratamento de doenças autoimunes, e no campo da terapia gênica. ${ }^{3}$

Trata-se de uma gordura solúvel derivada dos fungos Tolypocladium inflatum gams ${ }^{4,5}$ e, Beauveria nívea, sob a forma de polipeptídio cíclico composto por 11 aminoácidos $^{6}$. É um undecapeptídeo cíclico, lipofílico neutro de peso molecular $1202 \mathrm{Da}$ e com solubilidade aquosa muito baixa $\left(0,04 \mathrm{mg} / \mathrm{ml}\right.$ a $\left.25^{\circ} \mathrm{C}\right) .{ }^{7}$ Foi introduzida em 1978, para o tratamento dos transplantes alogênicos. ${ }^{5}$

Sua absorção quando administrada por via oral é incompleta e depende de cada paciente, sua metabolização é principalmente hepática, porém também é absorvida em pequenas quantidades no trato gastrointestinal e nos rins. Sua excreção é biliar e 6\% da dose inicial é excretada na urina sob forma de metabólitos. Possui meia vida de 8,4 horas e pico de concentração entre 1,5 a 2 horas após administração. Apenas $0,1 \%$ da CsA é excretada de forma pura na urina, nem mesmo a diálise pode facilitar sua excreção. Overdoses de CsA provocam êmese 
forçada duas horas após sua ingestão, podendo ocorrer hepatotoxicidade e nefrotoxicidade transitória. Dosagens muito altas provocaram morte na maioria dos episódios. ${ }^{6}$

O uso de CsA necessita de determinações periódicas das concentrações sangüíneas para monitorizar a dose terapêutica, com a finalidade de obter o efeito imunossupressor ideal, reduzindo os riscos de rejeição devidos à subdose do medicamento, ou toxicidade causada pelo excesso de medicamento no organismo. ${ }^{8}$ Existem atualmente no mercado várias técnicas disponíveis para dosagem de CsA sangüínea. A cromatografia líquida de alto desempenho (bigh performance liquid chromatographic - HPLC), a primeira técnica utilizada, fornece resultados bastante precisos e específicos, porém é de difícil automatização, com custo elevado e procedimentos trabalhosos ${ }^{8}$. A técnica de radioimunoensaio (radioimmunoassay - RIA) foi descrita pela primeira vez em 1981, na época, representou um avanço importante, porém exigia equipamento específico e pessoal técnico especializado para manuseio do material radioativo.

A CsA tem grande utilização em humanos ${ }^{9,10}$ para prevenção da doença do enxerto contra o hospedeiro (GVHD) onde foi utilizada em dosagens de 12 a $15 \mathrm{mg} /$ $\mathrm{kg} / \mathrm{dia}$; em cães, em doses de $15 \mathrm{mg} / \mathrm{kg}$ por via oral duas vezes ao dia durante os trinta e cinco primeiros dias, diminuindo para $7,5 \mathrm{mg} / \mathrm{kg}$ também por via oral duas vezes ao dia por mais trinta dias ${ }^{11}$, sendo em alguns episódios a causadora de rejeição de alguns enxertos ${ }^{12}$. Doses baixas dos fármacos induzem tolerância imune. ${ }^{13} \mathrm{~A}$ CsA vem sendo utilizada na Medicina Veterinária em inúmeras situações. . $^{11,14}$

Uma das questões importantíssimas relacionadas ao uso da CsA é o estabelecimento da dose que possa resultar em imunossupressão adequada ${ }^{14}, \operatorname{com} \mathrm{o}$ mínimo de efeitos colaterais, para garantia do sucesso dos transplantes. Na literatura são descritos diversos estudos realizados em cães, utilizando dosagens de $5 \mathrm{mg} / \mathrm{kg}$ e de
$7,5 \mathrm{mg} / \mathrm{kg}$, por via intravenosa, uma vez ao dia ${ }^{15}$; alguns não revelaram sua dose ideal, porém afirmam a manutenção de concentração sangüínea de 400 a $800 \mathrm{ng} / \mathrm{mL}$, objetivando o sucesso do transplante ${ }^{16}$.

As doses do fármaco variam conforme os estudos anteriores; temos na literatura dosagens de $5 \mathrm{mg} / \mathrm{kg}$ a cada doze horas, por via oral, com modificações subseqüentes, objetivando concentração sangüínea no valor de $250 \mathrm{ng} / \mathrm{mL}^{17}$; e recomendações que os níveis de concentrações séricas no período de 12 horas entre 1 administração e outra fiquem em aproximadamente $500 \mathrm{ng} / \mathrm{ml}$. durante os primeiros trinta dias, para então serem reduzidos para $250 \mathrm{ng} / \mathrm{mL} .{ }^{18,19}$

Em outro estudo utilizaram CsA em doses de $20 \mathrm{mg} / \mathrm{kg} / \mathrm{dia}$, associaram a mesma a azatioprina e predinisona, mensuraram os valores séricos de CsA periodicamente mantendo a concentração entre 400 e $500 \mathrm{ng} / \mathrm{mL}^{20}$; outro ainda, utilizaram CsA $10 \mathrm{mg} / \mathrm{kg} / \mathrm{a}$ cada 12 horas nos cinco primeiros dias e $15 \mathrm{mg} / \mathrm{kg} / \mathrm{a}$ cada 12 horas do sexto ao quadragésimo quinto dias. $^{21}$

\section{Material e Método}

O projeto proposto foi conduzido após aprovação da Comissão de Bioética da Faculdade de Medicina Veterinária e Zootecnia da Universidade de São Paulo (FMVZ-USP). Foram utilizados no experimento quatro cães da raça Golden Retriever normais todos com dois meses de idade, e quatro afetados pela distrofia muscular, (dois deles com dois meses um com cinco e outro com dez meses de idade), pertencentes ao grupo de animais do canil GRMD Brasil localizado no Departamento de Cirurgia da FMVZ-USP.

Foram considerados animais afetados pela distrofia muscular os cães Golden Retriever do sexo masculino submetidos ao exame de genotipagem, através da extração de DNA genômico do sangue de cordão umbilical, realizado pelo Centro de Estudos do Genoma Humano, da Universidade de 
São Paulo. O exame compreende na identificação da mutação de ponto, do sitio 3' de splice, do intron 6 do gene da distrofina. Essa mutação leva à perda do exon 7 durante o processamento do mRNA gerando um stop códon do exon 8 e um peptídeo com 5\% do tamanho normal da proteína distrofina. Cães considerados normais partem do mesmo processo de exame de genotipagem, porém sem constar a mutação a que se refere à doença.

Os animais GRMD receberam ciclosporina A e foram submetidos a transplante de células tronco de cordão umbilical. Os animais GRN receberam somente tratamento com ciclosporina A. O imunossupressor empregado teve sua dosagem variando entre $1 \mathrm{mg} / \mathrm{kg}$ e $10 \mathrm{mg} /$ $\mathrm{kg}$, a cada doze horas, por um período de 32 dias para os cães normais e de até 67 dias para os transplantados. As doses foram ajustadas, caso a caso, ao longo do tratamento na tentativa de manter concentrações séricas variando entre 250 e $500 \mathrm{ng} / \mathrm{L}$.

O transplante celular (somente para os G-GRMD) foi realizado pela equipe do IB-USP e da FMVZ-USP, de acordo com protocolo estabelecido em projeto próprio. No dia um do experimento, antes de qualquer administração de CsA ou do transplante, foram realizadas todas as avaliações propostas e seus resultados considerados como valores basais (controle). Ao longo do período de tratamento, iniciado no dia dois do experimento (duração de 32 ou de até 67 dias) foram avaliadas as concentrações séricas da CsA

Em intervalos de dois ou no máximo três dias, amostras de sangue de cada animal eram coletadas após doze horas da última administração de CsA, antes da nova administração do fármaco, por meio de punção da veia jugular ou cefálica em volume de aproximadamente $2 \mathrm{~mL}$, e acondicionadas em tubos contendo o anticoagulante ácido etileno diaminotetracético (EDTA) a 10\%. Em seguida eram encaminhadas ao Laboratório de Análises
Bioquímicas do Hospital Israelita Albert Einstein para dosagem, por meio da técnica de imunoensaio monoclonal denominado fluorescence polarization immunoassay - FPIA. Todos os animais recebiam o fármaco às 8 horas e às 20 horas diariamente.

\section{Resultados}

As concentrações séricas de ciclosporina, considerando cada animal individualmente, oscilaram ao longo do estudo. Foram observados picos muito altos em seis dos oito animais. Apenas o animal número 5 do grupo GRMD manteve as concentrações séricas entre $250 \mathrm{ng} / \mathrm{dL}$ e 500 $\mathrm{ng} / \mathrm{dL}$ ao longo do período de tratamento. O animal número 6, do mesmo grupo, não chegou a alcançar as concentrações séricas desejáveis. Em função dos resultados apresentados para cada animal, ao longo do tempo de tratamento, ajustes da dose de CsA foram feitos, respeitando os limites propostos, com objetivo de controlar as concentrações séricas do fármaco. Os resultados descritos podem ser visualizados na figura 1.

No que se refere aos efeitos colaterais relacionados ao tratamento com $\mathrm{CsA}$, todos os animais apresentaram diminuição do apetite de intensidade variável ao longo do período, mas sem gravidade. Um cão do grupo GRMD (número 8) apresentou reação de toxicidade à CsA caracterizada por vários episódios de vômitos e diarréia durante um período de 12 horas. Outro, dos animais do grupo GRMD (número 5), desenvolveu descamação cutânea intensa, gengivite e periodontite durante o tratamento imunossupressor.

\section{Discussão}

O estabelecimento de uma dose ideal de CsA é primordial para garantir a ação de imunossupressão a fim de evitar a rejeição de enxertos ${ }^{14}$ para tratamento de seres humanos ${ }^{11}$ e camundongos. No estudo aqui apresentado foram utilizadas doses que variaram de $1 \mathrm{mg} / \mathrm{kg}$ a $10 \mathrm{mg} / \mathrm{kg}$ a cada doze 
GRN 1

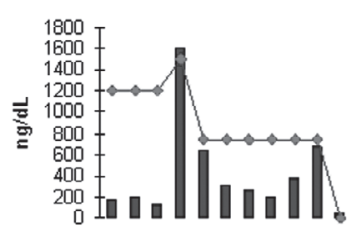

GRN 2

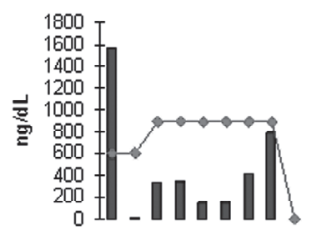

GRN 3

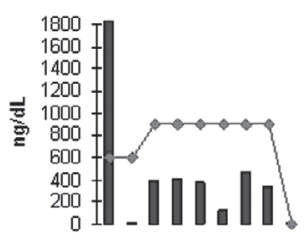

GRN 4

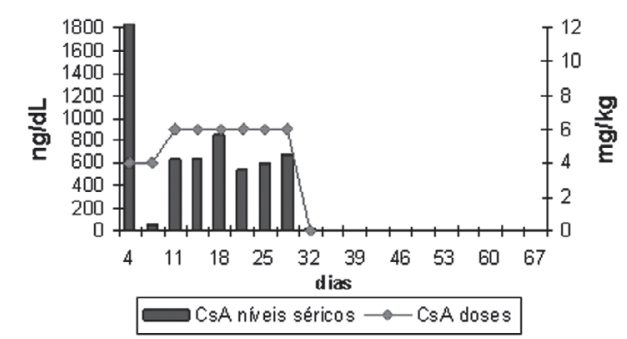

GRMD 5

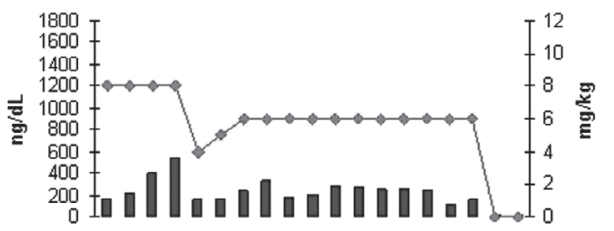

GRMD 6

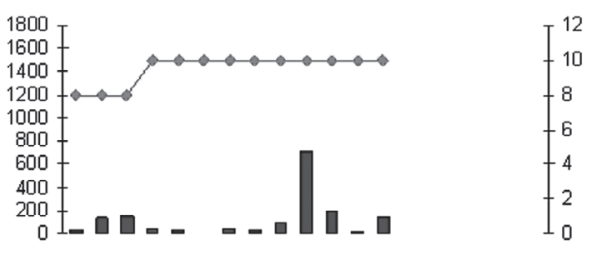

GRMD 7

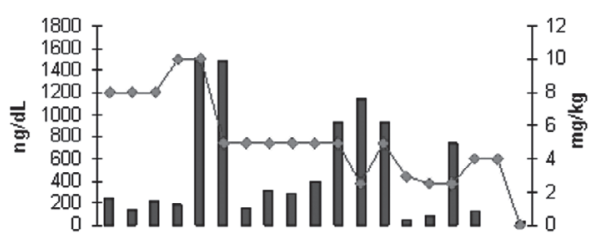

GRMD 8

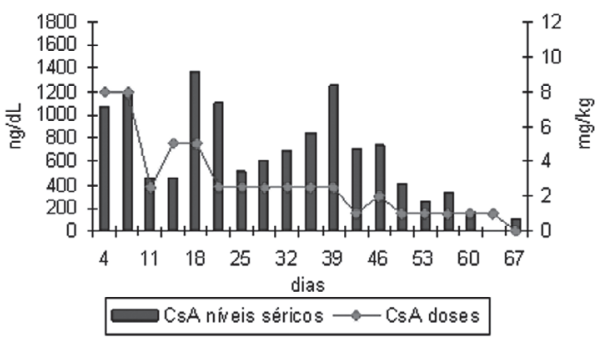

Figura 1 - Representações gráficas individuais das concentrações séricas de ciclosporina A em cães Golden Retriever normais ou afetados pela distrofia muscular progressiva submetidos ao protocolo de imunossupressão com ciclosporina A durante o período de 32 dias, nos cães normais (GRN) e 67 dias nos com distrofia muscular (GRMDs) - São Paulo - 2005

horas, valores esses semelhantes aos descritos para humanos ${ }^{10}$, para cães ${ }^{15,17,20,21,22}$, e também para gatos ${ }^{19}$. Em outros estudos realizados em cães foram empregadas doses maiores $13,18,19,20,23$.

Para ser obtido o efeito de imunossupressão, mais importantes que as doses do fármaco são as concentrações plasmáticas mantidas durante as 12 horas de intervalo entre as administrações. ${ }^{24}$ Nos tratamentos imunossupressores feitos com CsA, são consideradas como concentrações plasmáticas efetivas valores entre $250 \mathrm{ng} / \mathrm{dL}$ e $500 \mathrm{ng} / \mathrm{dL}^{17,20}$ para cães, para gatos ${ }^{18,19}$. Entretanto, outro estudo ${ }^{16}$ sugere que para cães, as concentrações séricas da CsA devem ser mantidas entre 400ng/dL e 800ng/dL. Estabeleceu-se como meta para o estudo aqui apresentado, a manutenção de concentrações séricas variando entre 250ng/ 
dL e 500ng/dL. Entretanto, houve variações nas concentrações séricas com ocorrências de picos que superavam mesmo aqueles do padrão preconizado pelos autores ${ }^{16}$ apesar de terem sido administradas doses baixas. Tais achados podem refletir particularidades da raça e idade dos animais estudados e, possivelmente, interferência da condição mórbida no caso dos cães GRMD.

$\mathrm{O}$ fato das concentrações séricas permanecerem oscilantes durante os primeiros meses justifica a indicação de um controle rígido das mesmas através de amostragens sanguíneas duas vezes por semana, que conforme o passar dos meses e a estabilização da concentração tornamse mais espaçados, chegando até a mensuração apenas anual, assim como descrito em noutro estudo. ${ }^{18}$

São relatados casos de óbito em animais de experimentação (camundongos, ratos e coelhos) em decorrência de concentrações séricas de CsA que ultrapassaram os limites seguros. ${ }^{25}$ Contudo, os filhotes de Golden Retriever estudados chegaram a apresentar concentrações séricas muito elevadas, com picos de até $1800 \mathrm{ng} / \mathrm{dL}$, sem alterações consideráveis da homeostase.

Embora estudos façam referências às vias de administração da CsA para humanos e animais ${ }^{20,21,25}$ não foram encontradas indicações específicas para pacientes afetados pela distrofia muscular. $\mathrm{O}$ fato do fármaco ter sido administrado por via oral, sob a forma de emulsão, facilitou o tratamento, principalmente dos cães GRMD os quais apresentam dificuldade em deglutir sólidos. A absorção também foi efetiva em pelo menos sete dos oito animais estudados como evidenciado pelas concentrações séricas detectadas.

\section{Conclusões}

Para tratamento imunossupressor efetivo para cães, as doses de CsA requerem ajuste orientado pelos valores séricos alcançados. Podem ser requeridos tanto aumentos como diminuições de doses ao longo de um tratamento. A idade dos cães é um fator importante para a determinação da dose apropriada de CsA. O Golden Retriever com idade inferior a 4 meses apresenta concentrações séricas perigosamente altas com doses que não causam o mesmo problema em animais com mais idade.

\section{Agradecimento}

À Fundação de Amparo a Pesquisa de São Paulo pelo apoio financeiro.

\section{Analyses of dosages and blood concentration of cyclosporin A on norrmal Golden Retriever dogs or Golden Retriever with muscular dystrophy}

\begin{abstract}
The muscular dystrophy of Golden Retriever (GRMD) is a degenerative miopaty caused by the absence of dystrophy and it is genetically homologue of the Duchenne muscular dystrophy in humans, so, these dogs are considerably experimental models for studies on cellular therapy. Their successful depends of the adequate immunosuppression. Cyclosporin A (CsA) is indicated for that and the monitoring of the blood concentration and adverse effects are essential to viabilise the therapy. It was studied GRMD dogs, and normal dogs from the same breed, submitted for therapy with CsA, associated, on GRMD, of cell transplantation. It was evaluated blood concentration of the drug, between two or tree days using the method of FPIA. In our results we found that the CsA blood concentrations oscillated too much on six than eight of our animals. We concluded that the doses varieties
\end{abstract}

Key words: Immunosuppression. Golden Retriever muscular dystrophy. Blood concentration. Fluorescence polarization immunoassay (FPIA). 
individually and the correct dosage as to important as the evaluation of the blood concentration of the drug and became viable for cell therapy.

\section{Referências}

1 BERGMAN, R. L.; INZANA, K. D.; MONROE, W. E.; SHELL, L. G.; LIU, L. A.; ENGVALL, E.; SHELTON, G. D. Dystrophin-deficient muscular dystrophy in a Labrador retriever. Journal of American Hospital Association, v. 38, n. 3, p. 255-261, 2002.

2 BOGDANOVICH, S.; PERKINS, K. J.; KRAG, T. O. B.; KHURANA, T. S. Therapeutics for Duchenne muscular dystrophy: current approaches and future directions. Journal of Molecular Medicine, v. 82, n. 2, p. 102-115, 2004.

3 CHILDERS, M. K.; OKAMURA, C. S.; BOGAN, D. J.; BOGAN, J. R.; PETROSKI, G. F.; MCDONALD, K.; KORNEGAY, J. N. Eccentric contraction injury in dystrophic canine muscle. Archieve of Physical Medicine Rehabilitation, v. 83, p. 1572-1578, 2002.

4 KORNEGAY, J. N.; BOGAN, D. J.; BOGAN, J. R.; CHILDERS, M. K.; CUNDIFF, D. D.; PETROSKI, G. F.; SCHUELER, R. O. Contraction force generated by tarsal joint flexion and extension in dogs with Golden Retriever muscular dystrophy. Journal of the Neurological Sciences, v. 166, n. 2, p. 115-121, 1999.

5 LIU, J. M. K.; OKAMURA, C. S.; BOGAN, D. J.; BOGAN, J. R.; CHILDERS, M. K.; KORNEGAY, J. N. Effects of prednisone in canine muscular dystrophy. Muscle \& Nerve, v. 30, n. 6, p. 767-773, 2004.

6 NGUYEN, F.; GUIGAND, L.; GOUBAULTLEROUX, I.; WYERS, M.; CHEREL, Y. Microvessel density in muscles of dogs with Golden Retriever muscular dystrophy. Neuromuscular Disorders, v. 15, n. 2, p. 154-163, 2005.

7 SHELTON, G. D.; LIU, L. A.; GUO, L. T.; SMITH, G. K.; CHRISTIANSEN, J. S.; THOMAS, W. B.; SMITH, M. O.; KLINE, K. L.; MARCH, P. A.; FLEGEL, T.; ENGVALL, E. Muscular dystrophy in female dogs. Journal Veterinary Internal Medicine, v.15, n. 3, p. 240-244, 2001.

8 MARQUES, M. J.; LUZ, M. A. M.; MINATEL, E.; SANTO NETO, H. Muscle regeneration in dystrophic $m d x$ mice is enhanced by isosorbide dinitrate. Neuroscience Letters, v. 382, n. 3, p. 342-345, 2005

9 FIRST, M. R. Immunosupressive Agents and Their Actions. Transplantation Proceedings, v. 34, n. 5, p. 1369-1371, 2002.

10 BENSOUSSAN, D.; LE DEIST, F.; LATGERCANNARD, V.; GRÉGOIRE, M. J.; AVINENS, O.; FEUGIER, P.; BOURDON, V.; ANDRÉ-BOTTÉ, C.; SCHIMITT, C.; JONVEAUX, P.; ELIAOU, J. F.; STOLTZ, J. F.; BORDIGONI, P. T-cell immune constitution after peripheral blood mononuclear cell transplantation in complete DiGeorge syndrome. British Journal of
Haematology, v. 117, n. 4, p. 899-906, 2002.

11 GONÇALVES, P. F.; NOGUEIRA FILHO, G. R.; SALLUM, E. A.; SALLUM, A. W.; NOCITI JUNIOR, F. $\mathrm{H}$. Immunosuppressant therapy and bone loss in ligatureinduced periodontitis - a study in rats. Pesquisa Odontológica Brasileria, v. 17, n. 1, p. 46-50, 2003.

12 LIN-WANG, H. T.; MANRIQUE, R. Aplicação da técnica de imunoensaio enzimático de multiplicação (EMIT) para dosagem de ciclosporina na amostra de sangue absorvido em papel filtro. Jornal Brasileiro de Patologia e Medicina Laboratorial, v. 38, n. 1, p. 7-12, 2002.

13 GEORGES, E. G.; STORB, R.; THOMPSON, J. D.; YU, C.; GOOLEY, T.; BRUNO, B.; NASH, R. A. Adoptive immunotherapy in canine mixed chimeras after nonmyeloablative hematopoietic cell transplantation. Blood, v. 95, n. 10, p. 3262-3269, 2000.

14 PAPICH, M. G. Immunosuppresssive Drugs. In: ACVIM SEATTLE, 18., 2000, Seattle, WA. Proceeding...p. 72-74, 2000.

15 YILMAZ, Z.; SÖZÜER, E.; BANLI, O. Effect of Combination of Cyclosporine $A$ and Bromocriptine on Survival of Renal Alografts in Dogs. Transplantations Proceedings, v.30, n. 3, p. 839-841, 1998.

16 DUNN, T. B.; KUMINS, N. H.; HOLMAN, D. M.; RAOFI, V.; BLANCHARD, J.; GLIMER, T.; POLLAK, R.; BENEDETTI, E. Multiple Sequential Transplantation of Hepatocytes in the Dalmatian Dog Model. Transplantation Proceedings, v. 31, n. 1-2, p. 543-544, 1999.

17 KYLES, A. E.; GREGORY, C. R.; GRIFFEY, S. M.; BERNSTEEN, L.; PIERCE, J.; LILJA, H. S.; MORRIS, R. E. Immunosuppression with a combination of the leflunomide analog, FK778, and microemulsified cyclosporine for renal transplantation in mongrel dogs. Transplantation, v. 75, n. 8, p. 1128-1233, 2003.

18 GREGORY, C. R.; GOURLEY, I. M.; KOCHIN, E. J.; BROADDUS, T. W. Renal transplantation for treatment of end-stage renal failure in cats. Journal of American Veterinary Medicine Approaches, v. 201, n. 2, p. 285-291, 1992.

19 KATAYAMA, M.; McANULTY, J. F. Renal Transplantation in Cats: Techniques, Complications, and Immunosupression. Compendium on Continuing Education for the Practicing Veterinarian, v. 24, p. 874-882, 2002.

20 BERNSTEEN, L.; GREGORY, C. R.; KYLES, A. E.; GRIFFEY, S. M.; PATZ, J. Microemulsified cyclosporinebased immunosuppression for prevention of acute renal allograft rejection in unrelated dog preliminary experimental study. Veterinary Surgery, v. 32, n. 3, p. 
213-219, 2003.

21 CONG, Y.; LINSLEY, P.; SEIDEL, K.; SALE, G.; DEEG, J. H.; NASH, R.; STORB, R. Cytotoxic T lymphocyte antigen 4-immunoglobulin fusionprotein combined wiyh methotrexate/cyclosporine as graft-versus-hostdisease prevention in a canine dog leucocyte antigennonidentical marrow transplant model. Transplantation, v. 69 , n. 3, p. 450-454, 2000.

22 HOGAN, W. F.; LITTLE, M.; ZELLMER, E.; FRIEDETZKY, A.; DIACONESCU, R.; GISBURNE, S.; LEE, R.; KUHR, C.; STORB, R. Postgrafting immunosuppression with sirolimus and cyclosporine facilitates stable mixed hematopoietic chimerism in dogs given sublethal total body irradiation before marrow transplantation from DLA-Identical littermates. Biology of Blood and Marrow Transplantation, v. 9, n. 8, p. 489-495, 2003.

23 SANDMAIER, B. M.; FUKUDA, T.; GOOLEY, T.; YU, C.; SANTOS, E. B.; STORB, R. Dog leukocyte antigen-haploidentical stem cell allografts after antiCD44 therapy and reduced-intensity conditioning in a preclinical canine model. Experimental Hematology, v. 31, n. 2, p. 168-175, 2003.

24 MCANULTY, J. F.; LENSMEYER, G. L. Comparison of high performance liquid chromatography and immunoassay methods for measurement of cyclosporine A blood concentrations after feline kidney transplantation. Veterinary Surgery, v. 27, n. 6, p. 586595, 1998.

25 NOVARTIS PHARMA. Neoral $^{\circledR}$. Soft geltin capsules. Neoral - cyclosporine. Apresenta dados de literatura sobre o imunossupressor ciclosporina $\mathrm{A}$, texto técnico científico. Disponível em: <www.pharma.us.novartis.com/product/pi/pdf/ neoral.pdf $>$. Acesso em: 14 fev. 2004. 